Nephrologe $2021 \cdot 16: 386-390$

https://doi.org/10.1007/s11560-021-00495-1

Angenommen: 8. Februar 2021

Online publiziert: 5. März 2021

(c) Springer Medizin Verlag GmbH, ein Teil von Springer Nature 2021

\section{Redaktion}

G. Wolf, Jena

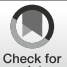

Jan-Hendrik Riedel' · Thorsten Wiech ${ }^{2}$ - Christian Krebs' · Ulf Panzer ${ }^{1}$

'Sektion für Translationale Immunologie, III. Medizinische Klinik und Poliklinik, Universitätsklinikum Hamburg-Eppendorf, Hamburg, Deutschland

${ }^{2}$ Sektion für Nephropathologie, Institut für Pathologie, Universitätsklinikum Hamburg-Eppendorf, Hamburg, Deutschland

\title{
Kreatininanstieg, Fieber und Hämoptysen
}

\section{"Double trouble"}

\section{Paraklinische Aufnahmebefunde}

\section{Anamnese}

Eine 25-jährige Patientin wurde aus einem externen Krankenhaus zur weiteren Diagnostik und Therapie bei seit 3 Wochen bestehenden Hämoptysen und febrilen Temperaturen mit Abgeschlagenheit und sukzessivem Anstieg des Serumkreatinins und der Inflammationsmarker trotz intravenöser antibiotischer Therapie übernommen. Es bestanden keine Vorerkrankungen bei insbesondere immer normwertigem Serumkreatinin und keine rezenten Auslandsaufenthalte. Ambulant war zuvor eine erfolgslose 6-tägige antibiotische Therapie mit Amoxicillin/Clavulansäure bei nativradiologisch imponierender Lobärpneumonie des rechten Unterlappens und Ausschluss einer Infektion mit SARS-CoV-2 („severe acute respiratory syndrome coronavirus 2 ") erfolgt. Eine noch vor Verlegung auswärts bei (eumorpher) Mikrohämaturie und Kreatininanstieg durchgeführte Nierenbiopsie hatte nur Muskelgewebe ergeben.

\section{Klinischer Befund}

Die Patientin präsentierte sich bei Übernahme voll orientiert in leicht reduziertem Allgemeinzustand ohne (Tachy-)Dyspnoe unter Raumluft. Die körperliche Untersuchung war, abgesehen von milden Knöchelödemen und feinblasigen Rasselgeräuschen über der rechten Lunge, unauffällig.
Bei einer subfebrilen Temperatur von $38^{\circ} \mathrm{C}$ lag eine Sinustachykardie von 108 Schlägen pro Minute bei regelrechtem Blutdruck vor. Die Sauerstoffsättigung lag bei $93 \%$ unter Raumluft. Laborchemisch zeigten sich eine Hämoglobinkonzentration von $10,1 \mathrm{~g} / \mathrm{dl}$ sowie eine Leukozytenzahl von $14 \mathrm{Mrd} . / \mathrm{l}$ mit relativer Neutrophilie und Lymphopenie bei leicht erhöhten Thrombozyten. Im Plasma imponierte eine Konzentration an C-reaktivem Protein (CRP) von $247 \mathrm{mg} / \mathrm{dl} \mathrm{mit}$ einem Procaltinonin(PCT)-Wert von $0,6 \mu \mathrm{g} / \mathrm{l}$. Die Serumkreatininkonzentration betrug 2,0 mg/dl (eGFR [geschätzte glomeruläre Filtrationsrate]: $35 \mathrm{ml} / \mathrm{min} / \mathrm{m}^{2}$ Körperoberfläche (KÖF) gemäß Chronic Kidney Disease Epidemiology Collaboration [CKD-EPI]) bei einem Wert von $1,4 \mathrm{mg} / \mathrm{dl} 48 \mathrm{~h}$ vor Übernahme bei normwertigen Leberwerten und einer leicht erhöhten Laktatdehydrogenasekonzen- tration (LDH) von 290 U/l. Die Elektrolyte, der $\mathrm{pH}$-Wert und die Blutgase waren ausgeglichen. Die Urindiagnostik zeigte die vorbekannte Mikrohämaturie ohne sicheren Akanthozytenund Zylindernachweis bei nichtrelevanter Leukozyturie. Die Albuminurie im Spontanurin betrug $250 \mathrm{mg} / \mathrm{g}$ Kreatinin. leicht echoreich vergrößert ohne Hinweis auf fokale Läsionen oder Harnstau. Die Vena cava inferior zeigte eine milde Dilatation mit beginnend aufgehobener Atemvariabilität.

\section{Weiteres Prozedere}

Eine bei Aufnahme durchgeführte Computertomographie des Thorax zeigte rechtsseitig eine flächenhafte Verdichtung mit perifokalen Milchglastrübungen ohne hiläre Lymphadenopathie, vereinbar mit einer pulmonalen Beteiligung bei Vaskulitis oder einem ausgeprägten pneumonischen Infiltrat Sonographisch zeigten sich die Nieren

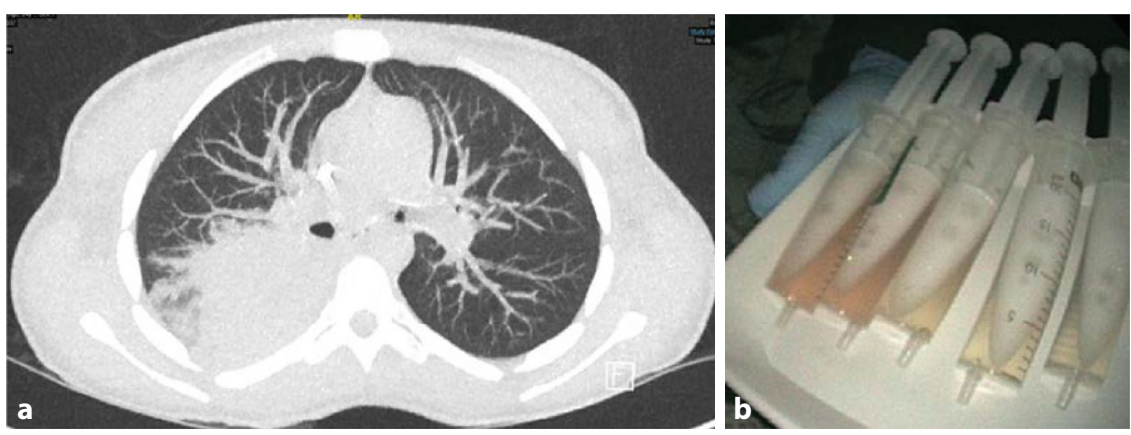

Abb. $1 \Delta$ Befunde der thorakalen Computertomographie (a) und der bronchoalveolären Lavage (b). (Mit freundlicher Genehmigung, @ Klinik für Radiologie [a] und Abteilung für Pneumologie [b], Universitätsklinikum Hamburg-Eppendorf [UKE]) 
Hier steht eine Anzeige.

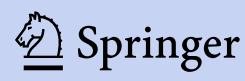



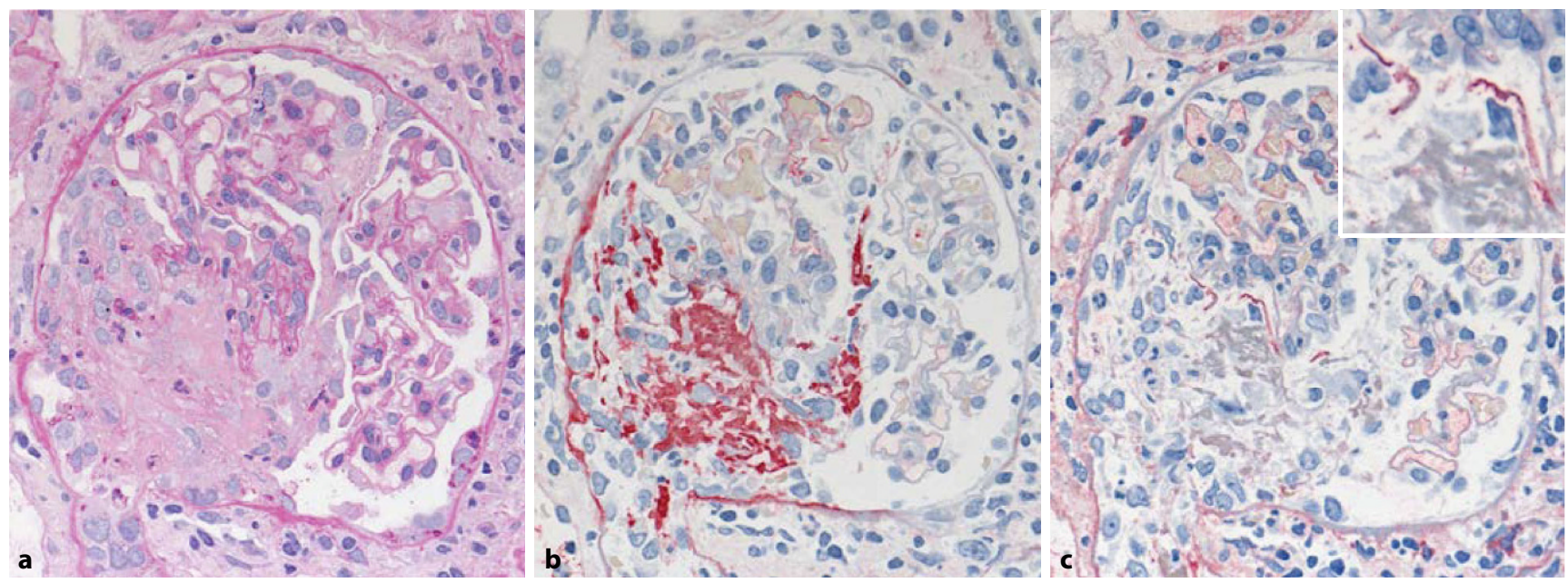

Abb. 2 \ Serielle Schnitte des Nierenbiopsats mit PAS(„,periodic acid-Schiff“; a)-, Fibrin- (b) und Immunglobulin-G(IgG)-Färbung (c; jeweils 400-fach mit Ausschnittvergrößerung in c)

(• Abb. 1a). Eine Bronchoskopie ergab eine akute, nichteitrige Bronchitis mit rechtsseitiger alveolärer Hämorrhagie in der bronchoalveolären Lavage (BAL; - Abb. 1b). Bei pulmorenalem Syndrom mit V.a. rapid-progressive Glomerulonephritis (RPGN) erfolgten zur Diagnosestellung eine erweiterte immunologische Diagnostik und eine erneute Nierenbiopsie. Bei Nachweis von hochtitrigen Antikörpern gegen die glomeruläre Basalmembran (GBM) sowie Proteinase 3 (PR3) erfolgten der umgehende Beginn einer hochdosierten immunsuppressiven Therapie mit intravenöser Gabe von Prednisolon und oraler Gabe von Cy- clophosphamid sowie der Beginn einer Plasmaaustauschtherapie unter Fortführung einer intravenösen Antibiotikagabe.

\section{Histologie}

In der Nierenbiopsie war lichtmikroskopisch eine herdförmig ausgebildete, überwiegend frisch nekrotisierende intra- und extrakapilläre Glomerulonephritis mit mittelschwerem, herdförmigem, potenziell teilweise reversiblem tubulointerstitiellen Begleitschaden der Rinde erkennbar. Von den 13 getroffenen Glomeruli waren in 7 frische Schlingennekrosen und extrakapilläre
Fibrinpräzipitate (•Abb. 2a, b) sowie in 1 Glomerulum ein fibrozelluläres Kapselproliferat erkennbar, kein Glomerulum war vollständig vernarbt. Der tubuläre Apparat war nur geringem Ausmaß herdförmig atrophisch. Immunhistologisch zeigten sich nur fokal und segmental lineare Ablagerungen von Immunglobulin $\mathrm{G}$ (IgG) an den Basalmembranen (- Abb. 2c) und keine glomerulären Ablagerungen von $\operatorname{IgA}, \operatorname{IgM}, \mathrm{Clq}$ oder C3. Elektronendichte mesangiale, subendotheliale oder subepitheliale Depots waren nicht vorhanden. Die Podozyten zeigten einen herdförmigen Verlust der Fußfortsätze.

\section{Wie lautet Ihre Diagnose?}

\section{Diagnose}

In der Zusammenschau der klinischen, immunologischen und histopathologischen Befunde ergab sich die Diagnose eines Overlaps aus Anti-GBM-Erkrankung und cANCA(antineutrophile zytoplasmatische Antikörper)-Vaskulitis (bei Zielantigen Proteinase 3 [PR3] formal Granulomatose mit Polyangiitis). Klinisch imponierte ein pulmorenales Syndrom mit akutem, progredientem Kreatininanstieg und im Verlauf abnehmender Diurese bei persistierender alveolärer Hämorrhagie und in dieser Kombination beginnender Oxygenierungsstörung. Immunologisch waren die Anti-GBM-Antikörper pathognomonisch für das Vorliegen einer AntiGBM-Erkrankung, die PR3-Antikörper wiesen auf das zeitgleiche Vorliegen einer cANCA-Vaskulitis hin. Die Nierenbiopsie bestätigte den Befund einer nekrotisierenden Glomerulonephritis mit Halbmonden. Da nicht alle Glomeruli betroffen waren, ist ein frühes Stadium der Erkrankung wahrscheinlich. Die hier allerdings nur fokal und segmental nachweisbare lineare Ablagerung von Immunglobulin (hier in der Regel $\operatorname{IgG}$ ) entlang der GBM ist pathognomonisch für die Anti-GBMErkrankung.

\section{Therapie und Verlauf}

Unter den oben beschriebenen Maßnahmen kam es bei fallenden Inflammationsmarkern initial noch zu einem weiteren Kreatininanstieg auf maximal 2,6 mg/dl (eGFR: $25 \mathrm{ml} / \mathrm{min} / \mathrm{m}^{2}$ KÖF nach CKDEPI) und bei persistierender Oligurie um $700 \mathrm{ml} /$ Tag 4 Tage nach Übernahme zur Notwendigkeit einer passageren Dialysetherapie für insgesamt 5 Tage zum Volumenentzug bei Zeichen der kardiopulmonalen Volumenüberladung und drohender respiratorischer Insuffizienz unter konservativer diuretischer Therapie. Nach 7 Tagen oraler Cyclophosphamidgabe erfolgte die erste intravenöse 
Gabe von $375 \mathrm{mg} / \mathrm{m}^{2}$ KÖF Rituximab zur Remissionsinduktion. 2 Wochen nach Übernahme und Beginn der hochdosierten immunsuppressiven Therapie und täglicher Plasmaaustauschtherapie konnte diese bei 2-malig negativ bestimmten Anti-GBM-Antikörpern, deutlich regredienten PR3-Antikörpern, fallendem Serumkreatinin und Sistieren der Hämoptysen beendet werden. Nach initialem Steroidpuls erfolgten ein orales Reduktionsschema in Anlehnung an die PEXIVAS-Studie [1] sowie die Vervollständigung der remissionsinduzierenden Therapie mit 3 weiteren Gaben von $375 \mathrm{mg} / \mathrm{m}^{2}$ KÖF Rituximab in wöchentlichen Abständen [2] mit seitdem im Serum stabil depletierten B-Zellen.

\section{》) Diagnose: Overlap aus Anti- GBM-Erkrankung und CANCA- Vaskulitis}

6 Monate nach Diagnosestellung zeigte sich die Nierenfunktion mit einem Serumkreatinin von 0,76 (eGFR: $110 \mathrm{ml} / \mathrm{min} / \mathrm{m}^{2}$ KÖF nach CKD-EPI) auf Normalniveau stabil. Es bestehen weder eine relevante Albuminurie noch andere pathologische Urinbefunde. Die AntiGBM- und die Anti-PR3-Antikörper lagen wiederholt unter der Nachweisgrenze der jeweiligen Tests. Eine remissionserhaltende Therapie mit $500 \mathrm{mg}$ Rituximab alle 6 Monate über mindestens 2 Jahre sowie eine niedrigdosierte Steroidtherapie mit $5 \mathrm{mg}$ pro Tag für 12 Monate mit anschließendem Tapering sind geplant.

\section{Diskussion}

Die Koexistenz von ANCA bei Patienten mit Anti-GBM-Erkrankung ist gut bekannt; in den vorliegenden Studien weisen etwa $30 \%$ der Patienten mit AntiGBM-Erkrankung nachweisbare ANCA auf, in der ganz überwiegenden Mehrheit gegen Myeloperoxidase (MPO), wohingegen bei etwa 5-10\% der Patienten mit ANCA-Vaskulitis Antikörper gegen die GBM detektiert werden können. Frühere Studien hatten bei jeweils geringen Fallzahlen diesen doppelt positiven Fällen widersprüchliche Verlaufsprognosen, zumeist jedoch einen ungünstigeren renalen Verlauf im Vergleich zu einer isolierten Anti-GBM-Erkrankung oder ANCA-assoziierten Vaskulitis (AAV; [3, 4]), zugeschrieben. Eine aktuelle multizentrische europäische Studie verglich die klinischen Merkmale und das Langzeit-Outcome von 37 doppelt positiven Patienten mit parallelen Kohorten von einfach positiven Anti-GBM-Erkrankungen $(n=41)$ und Patienten mit isolierter ANCA-Vaskulitis $(n=568$; [5]). Die doppelt positiven Patienten zeigten die schweren Krankheitsmerkmale einer Anti-GBM-Erkrankung mit hohen Raten von Alveolarblutungen (38\%) und initialer Dialysepflichtigkeit (57\%) mit einem Trend zur Erholung von der Dialyse bei den doppelt positiven $\mathrm{Pa}$ tienten im Vergleich zu den einfach positiven Anti-GBM-Erkrankten (33\% vs. $17 \%$ ). Darüber hinaus erlebten Patienten mit doppelt positiver Erkrankung während der Nachbeobachtung (Median: 4,8 Jahre) ein Rezidiv mit einer Häufigkeit, die derjenigen bei isoliert an ANCA-Vaskulitis Erkrankten entsprach, während keine einfach positiven AntiGBM-Patienten einen Rückfall erlitten. Diese Befunde weisen auf einen hybriden Krankheitsphänotyp mit den initial schweren Krankheitsmanifestationen der Anti-GBM-Erkrankung und dem langfristigen Rückfallrisiko der ANCAVaskulitis hin. Der Mechanismus dieser Assoziation zwischen ANCA-Vaskulitis und Anti-GBM-Erkrankung ist noch nicht vollständig verstanden. Der Umstand, dass doppelt positive Patienten die Altersverteilung von AAV-Patienten haben sowie die prodromalen Symptome und mehr chronische Läsionen in den Nierenbiopsien (Befunde, die eher typisch für ANCA-Vaskulitis sind) aufweisen, könnte darauf hindeuten, dass die ANCA-Vaskulitis der initiale pathologische Zustand ist, der die Anti-GBMErkrankung auslöst, indem die quaternäre Struktur der GBM zerstört und eigentlich sequestrierte Epitope für die Immunerkennung freigesetzt werden.

Im vorliegenden Fall ist eine mögliche zeitliche Abfolge der Erkrankungen nicht abschließend zu klären. Das Fehlen weiterer typischer Symptome/ Manifestationen einer cANCA-Vaskulitis sowie die im Nierenbiopsat überwie- gend frischen Nekrosen ohne Vernarbungen sprechen eher gegen eine AAV als Grunderkrankung. Der Umstand, dass im Biopsat nicht alle Glomeruli betroffen waren, und die nur fokale Positivität in der IgG-Färbung sind zwar eher untypisch für eine Anti-GBM-Erkrankung, könnten aber ein frühes Krankheitsstadium widerspiegeln. Ebenso spricht der Nachweis von PR3-Antikörpern gegen eine reine Anti-GBM-Erkrankung, da in der Regel fast ausschließlich MPOAntikörper bei doppelt positiven Patienten vorliegen. Erfreulicherweise zeigte die Patientin nicht nur eine bei doppelt positiven Antikörpern beschriebene teilweise Erholung der Nierenfunktion trotz intermittierender Dialysepflichtigkeit, sondern eine vollständige Normalisierung mit unauffälligen Urinbefunden.

\section{Fazit für die Praxis}

- Ein pulmorenales Syndrom mit V.a. zugrunde liegende Vaskulitis erfordert den umgehenden Beginn einer hochdosierten immunsuppressiven Therapie.

- Die Kombination aus laborchemischen, immunologischen und histopathologischen Befunden erlaubt in der überwiegenden Mehrzahl der Fälle eine schnelle Diagnose.

- Ein Overlap aus Anti-GBM(glomeruläre Basalmembran)-Erkrankung und ANCA(antineutrophile zytoplasmatische Antikörper)-Vaskulitis ist kein seltenes Phänomen und erfordert neben der umgehenden Therapieeinleitung die langjährige, engmaschige Nachsorge von Patienten mit ANCAVaskulitis.

\begin{tabular}{ll}
\hline Korrespondenzadresse \\
\hline \\
$\begin{array}{l}\text { Dr. Jan-Hendrik Riedel } \\
\text { Sektion für Translationale } \\
\text { Immunologie, Ill. Medizini- } \\
\text { sche Klinik und Poliklinik, } \\
\text { Universitätsklinikum } \\
\text { Hamburg-Eppendorf } \\
\text { Martinistr. 52, 20246 Ham- } \\
\text { burg, Deutschland } \\
\text { ja.riedel@uke.de }\end{array}$ \\
\hline
\end{tabular}




\section{Einhaltung ethischer Richtlinien}

Machen Sie sich fit mit dem „Facharzt-Training Innere Medizin“!

Interessenkonflikt. J.-H. Riedel, T. Wiech, C. Krebs und U. Panzer geben an, dass kein Interessenkonflikt besteht.

Für diesen Beitrag wurden von den Autoren keine Studien an Menschen oder Tieren durchgeführt. Für die aufgeführten Studien gelten die jeweils dort angegebenen ethischen Richtlinien. Für Bildmaterial oder anderweitige Angaben innerhalb des Manuskripts, über die Patienten zu identifizieren sind, liegt von innen und/oder ihren gesetzlichen Vertretern eine schriftliche Einwilligung vor.

\section{Literatur}

1. Walsh M, Merkel PA, Peh CA et al (2020) Plasma Exchange and Glucocorticoids in Severe ANCAAssociated Vasculitis. N Engl J Med 382:622-631

2. Stone JH, Merkel PA, Spiera Ret al (2010) Rituximab versus cyclophosphamide for ANCA-associated vasculitis. N Engl J Med 363:221-232

3. Levy JB, Hammad T, Coulthart A et al (2004) Clinical features and outcome of patients with both ANCA and anti-GBM antibodies. Kidney Int 66:1535-1540

4. Rutgers A, Slot M, Van Paassen P et al (2005) Coexistence of anti-glomerular basement membrane antibodies and myeloperoxidase-ANCAs in crescentic glomerulonephritis. Am J Kidney Dis 46:253-262

5. McadooSP, TannaA,HruskovaZetal (2017) Patients double-seropositive for ANCA and anti-GBM antibodies have varied renal survival, frequency of relapse, and outcomes compared to singleseropositive patients. Kidney Int 92:693-702
Bereiten Sie sich auf die Facharztprüfung vor oder möchten Sie Ihr fachspezisches Wissen mit typischen Fallbeispielen aus der Inneren Medizin auffrischen? Dann sind die Sonderhefte von Der Internist "FacharztTraining Innere Medizin" genau das Richtige für Sie.

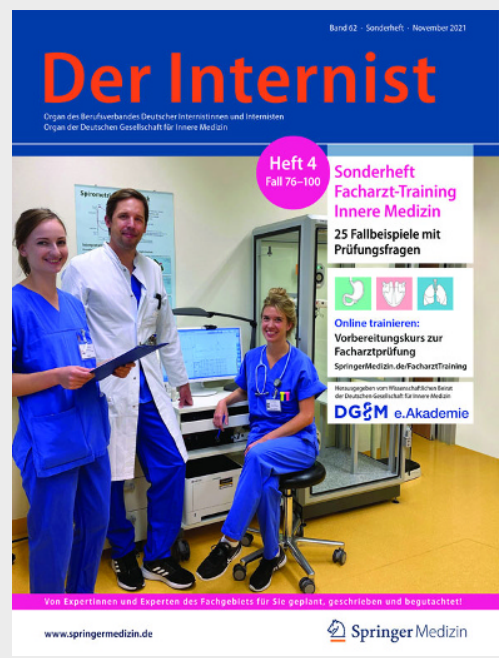

Sie finden in diesen Heften:

- Typische, alltagsnahe Fallbeispiele, systematisch und aktuell aufbereitet mit Prüfungsfragen und deren Antworten

- Kompaktes Wissen aus allen Fachbereichen der Inneren Medizin

- Sonderheft 1, 2, 3 und 4 sind erhältlich, weitere Sonderhefte folgen in Kürze

- Von Expertinnen und Experten für Sie geplant, geschrieben und begutachtet

- Herausgegeben vom wissenschaftlichen Beirat der Deutschen Gesellschaft für Innere Medizin

Bestellen Sie die Sonderhefte „FacharztTraining Innere Medizin" unter dem Aktionscode C0019640 bei

Marie-Luise.Witschel@springer.com. (1 Sonderheft 44 EUR, Paketpreis 2 Sonderhefte 75 EUR, Paketpreis 3 Sonderhefte 99 EUR, Paketpreis 4 Sonderhefte 125 EUR) 